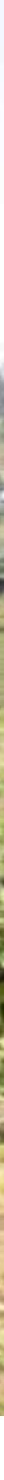

\title{
Guide de cartographie participative géoréférencée pour la gestion communautaire du terroir
}

Richard Sufo Kankeu

Anne Marie Tiani 



\section{Guide de cartographie participative géoréférencée pour la gestion communautaire du terroir}

Richard Sufo Kankeu

Centre de recherche forestière internationale (CIFOR)

Anne Marie Tiani

Centre de recherche forestière internationale (CIFOR) 
Document de travail 158

(c) 2014 Centre de recherche forestière internationale (CIFOR)

Le contenu de cette publication est soumis à une licence des Creative Commons Attribution 4.0 International (CC BY 4.0), http://creativecommons.org/licenses/by/4.0/

ISBN 978-602-1504-52-9

Sufo Kankeu R et Tiani AM. 2014. Guide de cartographie participative géoréférencée pour la gestion communautaire du terroir. Document de travail 158. Bogor, Indonésie : CIFOR.

Photo de Alba Saray Perez Teran/CIFOR

Paysage dans la cellule de Butaka, Rwanda

\section{CIFOR}

Jl. CIFOR, Situ Gede

Bogor Barat 16115

Indonésie

$\mathrm{T}+62(251) 8622-622$

$\mathrm{F}+62$ (251) 8622-100

E cifor@cgiar.org

\section{cifor.org}

Nous tenons à remercier tous les donateurs qui ont soutenu cette recherche avec leurs contributions au Fonds du CGIAR. Pour une liste des donateurs au Fonds, s'il vous plaît voir: https://www.cgiarfund.org/FundDonors

Tous les points de vue figurant dans cet ouvrage sont ceux des auteurs. Ils ne représentent pas forcément les points de vue du CIFOR, des responsables de la rédaction, des institutions respectives des auteurs, des soutiens financiers ou des relecteurs. 


\section{Table des matières}

Avant-propos

Liste des abréviations

1 Introduction

1.1 Définitions

1.2 Objectif de ce document

1.3 Approches

2 Les phases de la cartographie participative géoréférencée 3

2.1 Phase 1 : Préparation 3

2.2 Phase 2 : Collecte des données et numérisation 5

2.3 Phase 3 : Validation et finalisation de la carte 9

3 Les acteurs et le materiel $\quad 11$

3.1 Les acteurs de la CPGR 11

3.2 Matériel et outils $\quad 12$

4 Coût de la cartographie participative géoréférencée $\quad 14$

4.1 Matériel 14

4.2 La main d'œuvre 14

4.3 Le déplacement 14

$\begin{array}{llr}5 \text { Conclusion } & 17\end{array}$

6 Bibliographie $\quad 18$

Annexes

$1:$ Guide d'enquête $\quad 20$

2 : Liste indicative des éléments à représenter dans la légende $\quad 22$ 


\section{Liste des figures, tableaux et encadrés}

\section{Figures}

1

Les principales phases et étapes de la cartographie participative géoréférencée 3

$2 \mathrm{a}$ et $2 \mathrm{~b}$

Présentation du projet et de l'intérêt de la CPGR à Bompélo et à Mang Descente en forêt pour enregistrement des pistes et identification des types d'utilisation des terres

6 Carte participative sur papier (5a), intégration des premières données dans le SIG (5b) et le rendu après traitement et intégration des données dans un SIG (5c)

\section{Tableaux}

$1 \quad$ Format de fiche de collecte de données sur le terrain 6

2 Résultats attendus par étape 10

3 Liste détaillée de matériels de travail 13

4 Elément de coût du matériel de terrain pour la réalisation d'une carte participative géoréférencée 14

5 Dépenses effectuées lors de la phase terrain de la réalisation de la cartographie participative géoréférencée dans trois sites.

\section{Encadrés}

1 La mobilisation des acteurs de la CPGR en RDC 4

2 Importance de la connaissance du site $\quad 6$

3 Besoin de bien expliquer les objectifs de la CPGR 9

4 Se préparer à gérer les conflits $\quad 12$

5 Budgets des activités 16 


\section{Avant-propos}

Le projet 'Changement Climatique et Forêts dans le Bassin du Congo : Synergies entre l'Adaptation et l'Atténuation', COBAM en abrégé, a été initié par le CIFOR dans le cadre de l'aide accordée par la Banque africaine de développement $(\mathrm{BAD})$ à la Communauté économique des États de l'Afrique centrale (CEEAC), pour financer le Programme d'appui à la conservation des écosystèmes du Bassin du Congo (PACEBCo). Il a pour objectif de développer une réponse appropriée à la vulnérabilité liée aux changements climatiques dans les paysages du Bassin du Congo, en fournissant aux décideurs, praticiens et communautés locales les informations et outils nécessaires à la conception et à la mise en œuvre des politiques et des projets d'adaptation au changement climatique et de réduction des émissions de carbone dans les forêts, avec des impacts équitables et des co-bénéfices sur la réduction de la pauvreté, l'amélioration des services écosystémiques autres que le carbone, et la protection des modes d'existence et des droits locaux.

Le projet COBAM comprend deux volets que sont la recherche conventionnelle et la recherche-action dans des paysages de conservation. Ce dernier volet intègre cinq projets pilotes sur lesquels seront étudiés, entre autres, la vulnérabilité des populations locales, le rôle des forêts dans l'adaptation, le rôle joué par les activités de subsistance des populations sur la conversion des forêts ainsi que le suivi du stock de carbone dans des sites choisis. A cet effet, la stratification des paysages à partir des images satellitaires sera complétée par la cartographie participative géoréferencée sur deux périodes. Cette dernière approche a été retenue parce qu'elle permet de rendre fidèlement les informations et les intérêts des populations au niveau local tout en ayant une signification à l'échelle globale.

Ce document est un aide-mémoire. Il présente, sous forme d'un ensemble cohérent, une somme d'expériences acquises par les auteurs suite à une longue pratique de cartographie participative. La démarche proposée est celle utilisée dans le projet COBAM. Elle peut être appliquée telle quelle ou modifiée selon les situations. Elle peut servir aux facilitateurs et aux cartographes locaux pour élaborer des cartes du terroir, des ressources et de l'utilisation des terres dans le but d'initier des discussions communautaires. Elle peut aussi servir de référence aux chercheurs et aux praticiens qui voudraient encourager les communautés locales à spatialiser leurs activités et assurer le suivi du changement dans leur terroir. Ce document présente les étapes de la réalisation de la CPGR, les acteurs impliqués, le matériel et outils ainsi que les coûts. Dans le cadre du projet COBAM, c'est la première étape du processus de l'évaluation participative des stocks de carbone dans les sites de projets pilotes, et cela, avant leur mise en œuvre.

Les auteurs tiennent à remercier tous ceux qui ont de près ou de loin contribué à la rédaction de ce document. Plus spécialement Alba Saray Pérez Terán, Eugene Loh Chia et Louis-Bernard Cheteu pour leurs commentaires enrichissants ; les communautés d'Ekekam II dans la Région du Centre, de Yokadouma dans la Région de l'Est au Cameroun ; les populations de Lukolela dans la Province de l'Equateur en RDC et celles des Districts de Nyabihu et Rubavu de la Province de l'Ouest au Rwanda. 


\section{Liste des abréviations}

CPGR : Cartographie participative géoréférencée

COBAM : $\quad$ Changement climatique et Forêts dans le Bassin du Congo

CLIP : $\quad$ Consentement Libre Informé Préalable

FIDA : $\quad$ Fond international de développement agricole

GES : $\quad$ Gaz à Effet de Serre

GPS : $\quad$ Global Positioning System

MECNT: $\quad$ Ministère de l'Environnement de la Conservation de la Nature et du Tourisme

MINEPDED : Ministère de l'Environnement de la Protection de la nature du Développement Durable

ONG : $\quad$ Organisation non gouvernementale

P3DM : $\quad$ Maquette Participative en 3 Dimensions

PFNL : $\quad$ Produit Forestier non Ligneux

RDC : $\quad$ République Démocratique du Congo

RNRA : $\quad$ Rwanda Natural Ressource Authority

SIG : $\quad$ Système d'information Géographique

SIGP : $\quad$ Système d'information Géographique Participatif

USD : dollar Américain

UTM : $\quad$ Universal Transverse Mercator

WGS : $\quad$ système géodésique mondial 


\section{Introduction}

\subsection{Définitions}

La cartographie participative crée une opportunité concrète pour corriger et simuler dans une communauté une prise de conscience des problèmes de gouvernance, notamment en insistant sur les questions concernant la manière dont les intérêts de la communauté sont représentés (Anau et al., 2003). La cartographie participative est définie comme étant la création des cartes par les populations locales, souvent avec l'implication d'organisations d'appui, notamment des gouvernements (à différents niveaux), des organisations non gouvernementales (ONG), des universités et d'autres acteurs engagés dans le développement et la planification de l'accès aux terres (FIDA, 2009). Elle est devenue une méthode permettant d'incorporer le langage parlé dans une carte, dans le but, encore une fois, de donner corps aux voix de ceux qui sont en bas de l'échelle sociale, sur un support tangible et visible, facilitant le dialogue et les négociations. Elle représente aussi un outil commun et précieux utilisé pour permettre aux communautés d'enregistrer directement leurs limites, leurs types d'utilisation des terres et leurs ressources (Anau et al., 2003 ; Angeon et al., 2006). Elle implique la représentation par les populations locales et autochtones de leurs ressources, leurs zones d'activités, leurs lieux de cultes sous la supervision des autorités des ONG ou des acteurs de la société civile. Ainsi, toutes les catégories socioculturelles et ethniques sans discrimination doivent apporter leur expertise à la réalisation de cette carte (Kwaku Kyem, 2004; Jardinet, 2006). La cartographie participative permet de représenter les zones d'intérêts pour chaque groupe social ainsi que les ressources entrant dans leurs modes de vie. De ce fait, elle permet de prendre en compte ces aspects lors de la mise en place des projets pilotes. Toutes les lois et les standards environnementaux et sociaux recommandent la consultation préalable des populations locales et des peuples autochtones (CLIP) ou leur participation dans la gestion des ressources naturelles. La cartographie participative est un des outils les plus efficaces pour initier le dialogue entre les populations locales et les autres acteurs. Il est parfois utile de refaire une carte historique retraçant les traits des types d'utilisation des sols dans un passé récent ou lointain (Burini, 2005; Burini, 2008; Burini, 2013)
La cartographie participative géoréférencée (CPGR) est la réalisation de la carte par les parties prenantes en établissant la relation entre des coordonnées spatiales de la carte plan réalisée par les communautés et les coordonnées connues dans la réalité grâce aux technologies spatiales telles que les SIG (Systèmes d'Information Géographique), le GPS (Global positioning System) et la télédétection. Ces technologies offrent une promesse de renforcement des capacités locales pour la gestion des terres, la résolution des conflits et un planning proactif (PALSKY, 2010; Hervé et al., 2014). Les coordonnées prises au GPS après la réalisation de la carte participative permettent de positionner les objets et infrastructures à leur emplacement réel. La cartographie participative géoréférencée capitalise tous les avantages de la cartographie participative dans la mesure où elle peut être utilisée par différents groupes sociaux comme support de réflexion au sujet de la gestion des ressources naturelles communautaires. En plus, elle présente de nombreux autres atouts parmi lesquels :

- la précision dans la disposition sur la carte des centres d'intérêts, des zones d'activités et des distances

- une meilleure visualisation

- une plus grande fluidité dans la communication expert-communauté-politique

- un archivage plus sécurisé.

\subsection{Objectif de ce document}

Ce document n'a pas la prétention d'être un guide à proprement parler. Tout au plus, c'est une liste de contrôle qui voudrait mettre à la disposition des chercheurs, des praticiens ou des cartographes débutants, des informations de base sur la démarche à suivre pour réaliser des cartes participatives géoréférencées. Elle les aidera dans l'identification des acteurs, le choix des techniques et des outils nécessaires, les étapes à suivre et les coûts afférents.

Le document est divisé en trois sections. La première présente les acteurs, le matériel et les outils requis ; la deuxième section donne le détail des étapes de la réalisation de la cartographie, de lancement du projet 
à la validation de la carte; la troisième esquisse des éléments à prendre en compte lors du calcul du coût de l'opération.

\subsection{Approches}

Plusieurs méthodologies de cartographie participative ont vu le jour, de l'approche impliquant une participation massive de tous les villages, à celle incluant l'utilisation des SIG et de la télédétection (Chapin et al., 2005 ; Chambers, 2006). Elle est qualifiée de participative puisqu'elle mobilise le savoir et le savoir-faire des communautés locales et de tous les acteurs. La majorité des approches méthodologiques pour la réalisation de la cartographie participative préconise les étapes suivantes : la préparation de la communauté à l'activité de cartographie, la définition des objectifs du processus cartographique, la collecte des données, la création de la carte et de la légende, l'analyse et l'évaluation de l'information, l'utilisation et la communication des informations spatiales de la communauté (Chapin et al, 2005 ; PALSKY, 2010; Burini, 2013).

Il existe plusieurs types de cartographie participative :

- la cartographie pratique (au sol ou sur papier),

- la cartographie participative avec cartes à échelle et images (utilisation des images satellites et photos aériennes)

- les maquettes participatives en trois dimensions (P3DM)

- les systèmes d'information géographique participatifs (SIGP) qui intègrent le savoir local et les données qualitatives du SIG

- la cartographie multimédia et sur la toile, à savoir, la réalisation des cartes/ou ajouts des données par un groupe d'experts sur la toile, des vidéos, des données audio.

- $\quad$ Le wikimaps (PALSKY, 2010)
Dans le cadre du projet COBAM nous avons utilisé celle dite « cartographie participative géoréférencée (CPGR) » qui combine la carte pratique (sur papier) la cartographie participative avec des cartes à échelle et les SIG. Elle implique aussi l'utilisation des logiciels de traitement d'images et de cartographie, les images satellitaires, les GPS spécialisés, les photographies aériennes. La carte historique des localités concernées par le projet a été réalisée.

La CPGR a été choisie parce qu'elle permet d'impliquer toutes les couches de la population, y compris les analphabètes avec lesquels il faut travailler avec des supports visuels. Elle a aussi l'avantage de minimiser les coûts et de gagner en précision. Elle est non seulement pratique, mais aussi crédible, peu coûteuse, rapide et précise (PALSKY, 2010; Burini, 2013). Dans un contexte de forte dépendance technique, financière et politique, le déploiement de ce type d'approche pose avec acuité la question éthique et nécessite une réflexion sur la portée sociale des cartes présentées comme participatives (Amelot, 2013). La CPGR est un outil simple et approprié dans la mesure où elle favorise en même temps la réflexion collective et l'apprentissage mutuel.

Les objectifs de la CPGR sont variés car ils dépendent non seulement de celui qui l'a initiée, mais aussi du contexte et des objectifs initiaux du projet pour lequel elle est réalisée. Globalement, elle est utilisée pour poser les bases du suivi des ressources dans un territoire bien défini à travers un partage de connaissances de ces ressources, l'identification des acteurs, leurs interactions, leur mode d'utilisation des ressources, la visualisation de la dynamique de ces interactions ainsi que leurs impacts sur le couvert forestier. 


\section{Les phases de la cartographie participative géoréférencée}

Globalement, la cartographie participative géoréférencée comprend trois phases : la préparation, la collecte des données et leur numérisation, enfin la validation et la finalisation de la carte. Chacune de ces trois étapes se déroule alternativement au bureau et sur le site (voir figure 1).

\subsection{Phase 1 : Préparation}

La préparation de la CPGR se fait d'abord au bureau, puis sur le site.

\subsubsection{Préparation au bureau}

Il s'agit de rassembler toutes les données, les cartes et les outils pouvant faciliter le travail sur le terrain. Au préalable, il faut identifier la zone à cartographier ainsi que les villages concernés par le projet. Les éléments topographiques et cartographiques de chaque village seront identifiés et insérés dans les fonds de carte. Il s'agit des cours d'eau, des routes, des montagnes, des limites naturelles, etc. Des cartes de chaque village (à grande et à petite échelle) seront ainsi réalisées à partir des fonds topographiques, des images satellitaires et cela, à l'aide des logiciels de cartographie ou de télédétection. Ceux-ci peuvent être acquis gratuitement (Qgis) ou achetés (ENVI 5, ERDAS IMAGINE 2011 et Arcmap 10.1). Ces cartes serviront de base lors de la réalisation de la carte sur le terrain.

La carte à grande échelle permet d'avoir une vue d'ensemble du site du projet. Elle contient des éléments qui serviront de repères aux populations locales lors de l'élaboration de la CPGR. Elle présente aussi en détail les lieux, les routes, les cours d'eau et dans la mesure du possible l'habitat (pour les images satellites). La carte à petite échelle présente une vue sur la région et les activités environnantes au projet. Celle-ci montre non seulement les limites du projet, mais aussi les autres unités environnantes pouvant interagir avec le projet (ou constituant une zone de fuite).

Dans la mesure du possible, il faudra préparer la descente sur le terrain en fixant des rendez-vous et en établissant un calendrier de travail avec les organisations partenaires présentes dans le site. Les objectifs de la CPGR devraient être bien compris par

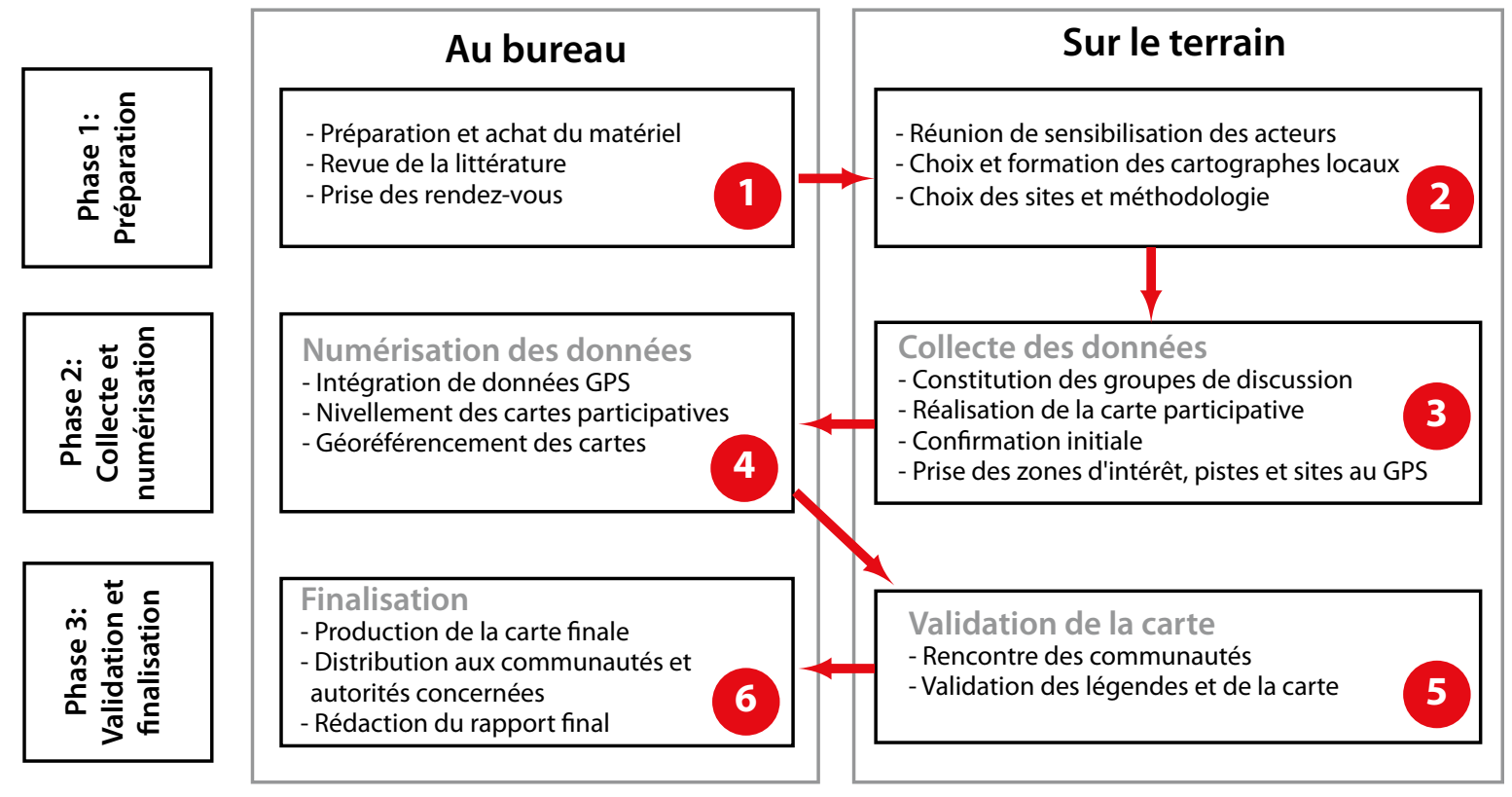

Figure 1. Les principales phases et étapes de la cartographie participative géoréférencée

Sources : Adaptés de Long et al. (2013) et FIDA (2010) 

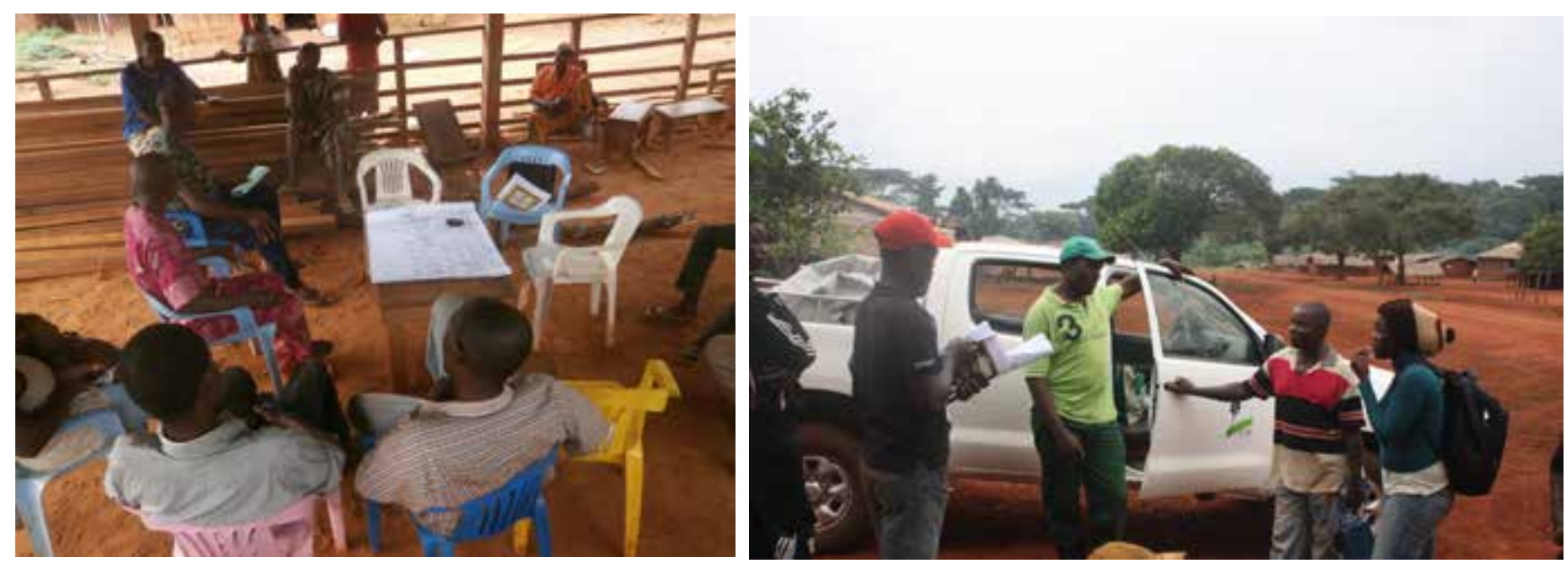

Figure 2a et 2b. Présentation du projet et de l'intérêt de la CPGR à Bompélo et à Mang (Photo : Richard Sufo Kankeu et Merline Touko)

\section{Encadré 1. La mobilisation des acteurs de la CPGR en RDC}

L'identification et la mobilisation des acteurs de la CPGR peuvent être plus ou moins aisées en fonction du contexte sociopolitique et de l'organisation sociale du pays. Le Rwanda est gouverné par une administration fortement centralisée et hiérarchique. II revient aux chefs de cellules et chefs de village de choisir les acteurs. Dans le cas de l'ouest de la RDC caractérisée par une structure de gouvernance locale plus lâche, quasi acéphale, les acteurs ont été identifiés et mobilisés par les experts, en accord avec les chefs traditionnels.

Si pour des raisons de légitimité, il est important de faire intervenir les autorités locales dans le choix des cartographes locaux, l'expérience montre que cette option présente de nombreux défis :

- Certaines autorités ont exigé des cartographes une partie de leur 'salaire'.

- D'autres n'ont sélectionné que les gens de leur entourage ou de leurs hameaux, ce qui a engendré des frustrations au sein des communautés.

- D'autres encore ont intentionnellement caché l'existence des groupes sociaux qui ne leurs sont pas inféodés.

Il est important de bien clarifier les critères de choix des cartographes locaux. La disponibilité, le sens du partage, la connaissance avérée de la vie au village ne suffisent pas toujours. L'équité en matière de genre ou la représentativité des groupes sociaux sont autant de critères à prendre en compte si on veut éviter des ressentiments.

les autorités administratives et traditionnelles ainsi que les personnes ressources à rencontrer.

Dans le cadre de la CPGR, des va-et-vient sont nécessaires entre le bureau et le terrain ; d'abord pour la sensibilisation des acteurs, puis pour la validation des cartes élaborées sur le terrain.

\subsubsection{Préparation sur le terrain}

Il s'agit ici de préparer les acteurs du site à accueillir l'équipe des experts. De ce fait, il faut rencontrer les autorités traditionnelles, administratives et religieuses, sensibiliser les populations cibles et identifier les cartographes.
Les rencontres avec les autorités administratives, traditionnelles et religieuses ont pour objectif la prise de contact, la sensibilisation aux objectifs à l'importance de l'exercice ou du projet. Les autorités sont contactées une par une, par une équipe du projet. Les autorisations et les lettres circulaires sont obtenues auprès des autorités concernées.

Des réunions ou rencontres de sensibilisation devront être tenues avec les différentes communautés dans le but de les informer sur la CPGR et sur les différents exercices qui seront effectués. 
L'identification des cartographes locaux peut être faite par les chefs de villages. Cependant, il faut pour cela mettre à leur disposition des critères de sélection bien précis afin de minimiser les subjectivités. Les cartographes locaux ainsi identifiés seront formés au début de la phase de collecte des données. Ils doivent appartenir aux deux sexes, être en bonne santé physique, instruits (savoir lire et écrire), jeunes (20-60 ans), être honnêtes et sociables. Ils doivent par ailleurs être résidents, connaitre les us et coutumes et l'environnement du village. Une fois les cartes schématisées, celles-ci doivent être validées par un groupe assez représentatif des communautés dont le terroir est cartographié.

La sensibilisation est primordiale, car elle va déterminer la qualité des résultats, et par conséquent, la réussite de l'exercice. Une fois les populations et les autorités mises en confiance, il conviendra d'établir avec elles le planning des activités de terrain. C'est également au cours de cette descente que les sujets potentiellement conflictuels pourront être décelés et une approche élaborée pour les aborder.

La communauté doit aussi décider des informations additionnelles à inclure dans la carte. Il peut s'agir de l'emplacement des éléments naturels (rivières, montagnes, pâturages), des constructions (villages, routes, zones agricoles), des ressources (types de forêts, zones de chasse ou de pâturage) ou des sites à valeur culturelle ou historique (frontières, cimetières ou zones d'importance spirituelle). Les zones de conflit potentiel, de changement d'utilisation des terres, de développement des activités à grande échelle doivent être recensées (Flavelle 2002)

\subsection{Phase 2 : Collecte des données et numérisation}

La phase de collecte des données et de numérisation débute par la formation des cartographes locaux qui seront déployés ensuite sur le terrain.

\subsubsection{Formation des cartographes}

La formation des cartographes comprend les modules suivants :

- Lecture de la carte et de la légende et l'utilisation du GPS,

- La notion d'échelle.

Le formateur doit éviter de frustrer les apprenants par des attitudes paternalistes ou condescendantes. Les séances de formation doivent être interactives, conviviales, avec des outils et méthodes accessibles. Le jour de l'atelier, les formateurs devront vérifier la disposition de la salle (rangée, disposition des participants). L'atelier s'ouvre généralement sur des exercices brise-glace visant la mise en confiance des participants comme la présentation individuelle, la danse ou des exercices physiques pouvant servir à cet effet (The Rainforest Foundation, 2013).

\subsubsection{Types de données à collecter}

Les données à collecter sont à la fois d'ordre qualitatif et quantitatif.

Les données qualitatives sont celles qui décrivent les couleurs, l'état des objets auxquels on ne peut pas attribuer de valeur. Elles sont collectées lors des échanges avec les populations locales et au cours de la réalisation des cartes participatives dans les groupes de discussion. Il s'agit de positionner sur les cartes tous les éléments marquants de l'environnement.

Les données quantitatives sont celles qui peuvent être mesurées. Elles sont collectées à l'aide des GPS et des fiches d'enregistrement. Il s'agit du nombre, de la longueur ou de la superficie et des coordonnées GPS des sites sacrés, des champs, des zones de chasse, des arbres à chenilles, des zones de pêche, des pistes...

Les données sont enregistrées dans des tableaux du même format que le tableau 1 .

\subsubsection{Réunion de sensibilisation avec les communautés}

Le cartographe ou le facilitateur explique la raison d'être de la réunion, le rôle attendu de chacun et les résultats. Les communautés doivent être informées du détail de la démarche qui sera suivie. Elles doivent savoir par exemple que les informations collectées seront reportées sur la carte. Enfin, les cartes générées sont à la fois la propriété des initiateurs et des communautés. De ce fait, une copie doit leur être remise à la fin.

\subsubsection{Réalisation de la carte participative géoréférencée}

La réalisation de la carte participative géoréférencée est un exercice interactif qui suscite beaucoup d'intérêt de la part des parties prenantes. Le rôle des experts externes se limite à la facilitation, qui se fait à l'aide de questions bien structurées. La durée moyenne d'un tel exercice est de trois à quatre heures. Pour plus d'efficacité, la carte participative doit être réalisée par un nombre réduit de personnes. Trois hommes et deux femmes de tous les âges, choisis 
Tableau 1. Format de fiche de collecte de données sur le terrain

\begin{tabular}{|c|c|c|c|c|}
\hline \multirow{3}{*}{$\begin{array}{l}\text { Nom ou } \mathrm{N}^{\circ} \mathrm{du} \\
\text { point }\end{array}$} & \multirow{3}{*}{ Description du point } & \multicolumn{2}{|c|}{ Coordonnées du point } & \multirow{3}{*}{$\mathbf{N}^{\circ}$ de la photo } \\
\hline & & \multicolumn{2}{|c|}{ Système projection : UTM, WGS 84, 35 S } & \\
\hline & & $x$ & $\mathrm{Y}$ & \\
\hline Site sacré & Arbre, rocher etc. & 520580 & 251030 & $\mathrm{~N}^{\circ} 5$ \\
\hline Zone de cueillette & $\begin{array}{l}\text { PFNL (Njansang, mangue, } \\
\text { marantacée) }\end{array}$ & 562010 & 201542 & $\mathrm{~N}^{\circ} 2$ \\
\hline \multicolumn{5}{|l|}{ Zone de chasse } \\
\hline \multicolumn{5}{|l|}{$\begin{array}{l}\text { Limites avec } Y \\
\text { village }\end{array}$} \\
\hline \multicolumn{5}{|l|}{ Cacaoyers } \\
\hline $\begin{array}{l}\text { Zone à risque } \\
\text { (inondation, }\end{array}$ & & & & \\
\hline
\end{tabular}

parmi les gens qui ont la réputation de bien connaitre le village. Les autres membres de la communauté doivent observer l'exercice et n'intervenir que lorsqu'ils sont sollicités. La validation de la carte se fera par la suite, par les autres membres de la communauté. Le protocole proposé est le suivant :

1. Le cartographe expert propose une légende qui sera examinée, complétée et validée.

2. Le cartographe expert prépare de grandes feuilles de papier devant servir à l'exercice.

3. Les limites du village et du projet sont dessinées sur le papier, ainsi que la route qui traverse le village. La zone de fuite potentielle est indiquée.

4. Les éléments immuables du paysage tels que les marécages, les cours d'eau, les montagnes, sont dessinés sur la carte.

5. Les aires d'habitation et les infrastructures sociales sont marquées.
6. Les jardins de case, les infrastructures et les mosaïques forestières sont représentés.

7. L'anthropologue-sociologue utilise les éléments de la carte pour la discussion des sujets de recherche.

Chaque fois que les acteurs sont appelés à disposer sur la carte/ou au sol des informations, activités et sites d'intérêt, l'information doit être confirmée par les autres membres de l'équipe. Il est par exemple demandé de disposer sur la carte les zones de chasse, de cueillette, de pêche ou d'agriculture, les cours d'eau (les nommer). Les positions, les formes et les proportions, la distance des objets sont vérifiées et confirmées par les autres membres du groupe. Les groupes spécifiques (groupe marginalisé ou genre) doivent être réunis pour spécifier et indiquer clairement leurs centres d'intérêts. Les informations

\section{Encadré 2. Importance de la connaissance du site}

Nous avons planifié la réalisation de la CPGR à Lukolela en RDC avant que la zone du projet ne soit circonscrite. Par conséquent, les deux activités ont été faites en même temps. C'est sur place que nous nous sommes rendus compte des difficultés de déplacement dans le site. L'accès à certains villages se faisait par pirogue motorisée et nécessitait deux jours de voyage fastidieux. Dans les villages, les gens n'étaient pas bien informés sur nos activités. Ils ont accepté de collaborer soit parce que la demande venait d'une autorité soit à cause des avantages pécuniaires y afférents, mais ils n'ont pas toujours donné la bonne information parce que le climat de confiance n'était pas établi.

Une bonne préparation de la CPGR requiert un va-et-vient entre le bureau et le terrain afin d'informer les autorités administratives et traditionnelles. II est tout aussi important d'effectuer des transects pour comprendre les réalités du terrain et les exigences physiques requises. Dans bien des cas et pour de nombreuses raisons dont les contraintes financières ou de temps, il n'a pas toujours été possible de suivre à la lettre toutes les étapes. Elles ont été sujettes à des modifications en fonction des difficultés rencontrées. Il est aussi important de se ménager une grande marge de manœuvre budgétaire qui permettrait de s'adapter à de nombreux imprévus qui ne manqueront pas de survenir sur le terrain. 

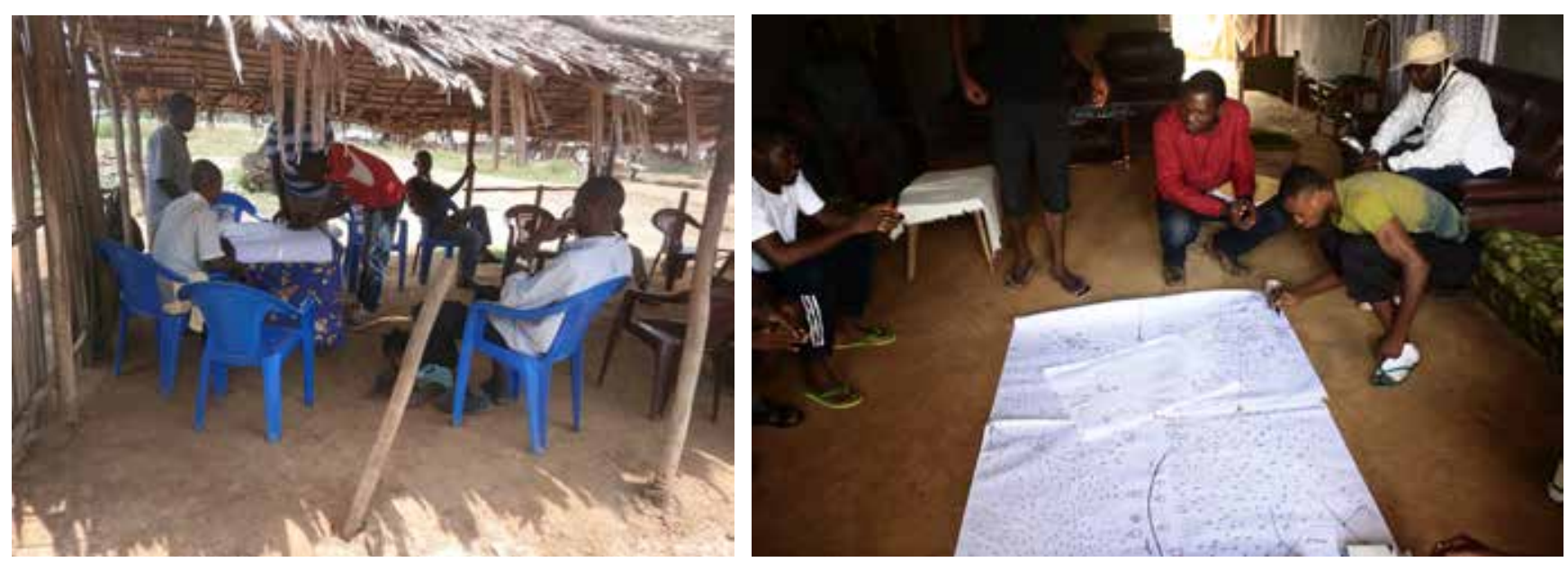

Figure 3a et 3b. Réalisation de la carte participative à Bongonda en RDC et à Ekekam Il au Cameroun (Photos : Richard Sufo Kankeu et Alba Saray Pérez Terán)
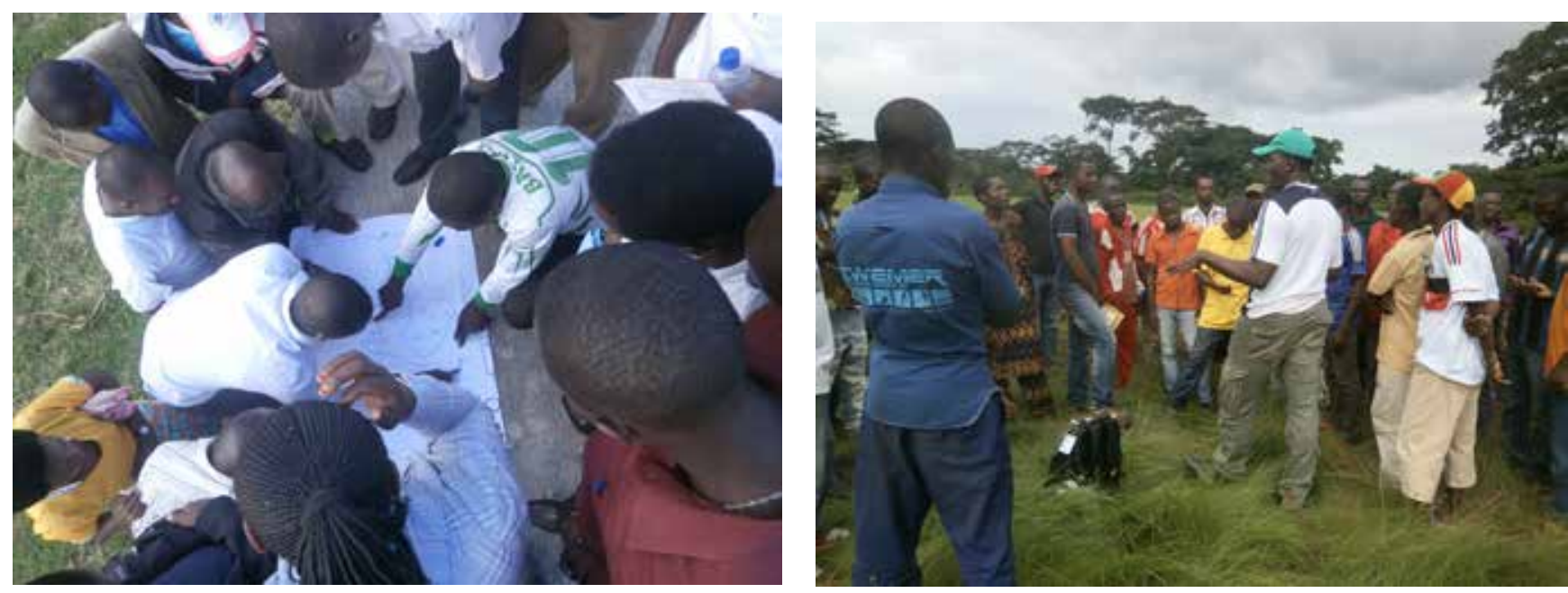

Figure 4a et 4b. Formation des cartographes locaux à la prise des coordonnées géographiques au GPS et à la réalisation des cartes dans le district de Nyabihu (Rwanda) et à Mang (Cameroun) (Photo : Richard Sufo Kankeu)

sont reportées sur la carte au fur et à mesure. Chaque participant est appelé à donner son avis sur le rendu final de la carte. La légende préparée doit être unanimement validée par les groupes de discussion (Rambaldi, 2005; Di Gessa, 2008). Afin d'éviter des conflits d'intérêts et les frustrations, des sous-groupes genre et peuples marginalisés sont organisés à part (Corbett and Rambaldi, 2009; Denniston, 1994). Les informations générées lors de l'élaboration de la carte participative sont au moins aussi importantes que cette carte elle-même. C'est l'occasion de collecter les informations sur les modes d'utilisation des différents écosystèmes et leur contribution à la sécurité alimentaire ou à la lutte contre la pauvreté. C'est également le lieu de discussion au sujet des droits d'accès, de la gouvernance, du fonctionnement des différentes institutions qui gouvernent la gestion des ressources naturelles (PALSKY, 2010; Burini, 2013). C'est enfin le moment de discuter de la vulnérabilité des toutes les entités présentes sur le site aux multiples stress, y compris climatiques. Les informations émanant des discussions doivent être consignées soigneusement et figurer dans le rapport. Le plus souvent, un traducteur est réquisitionné parmi les participants.

Le cartographe finalise la carte en demandant les informations complémentaires pour réduire les incompréhensions.

\subsubsection{Prise des coordonnées géographiques au GPS}

$\mathrm{Au}$ terme de ce travail préliminaire, les cartographes formés accompagnés des représentants des groupes 


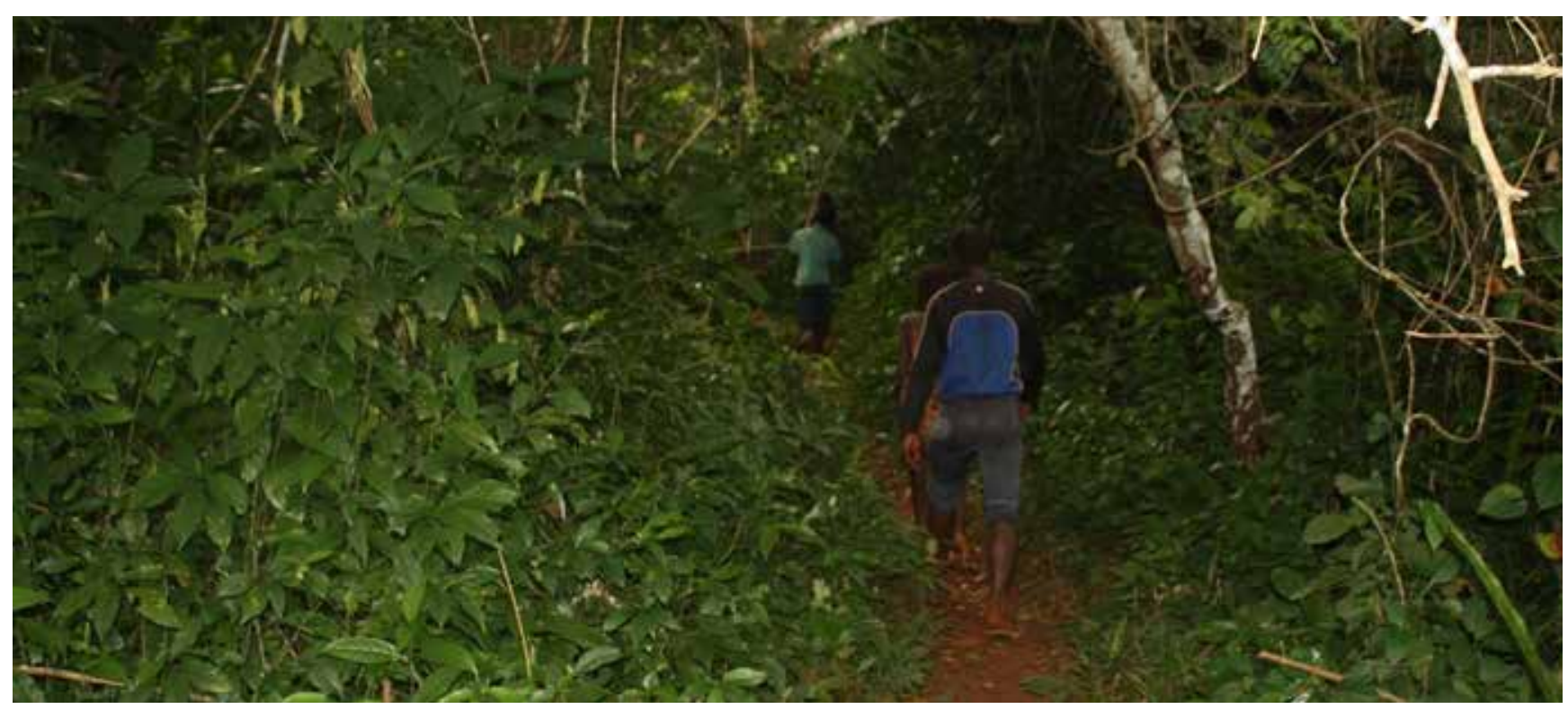

Figure 5. Descente en forêt pour enregistrement des pistes et identification des types d'utilisation des terres (Photo : Merline Touko)
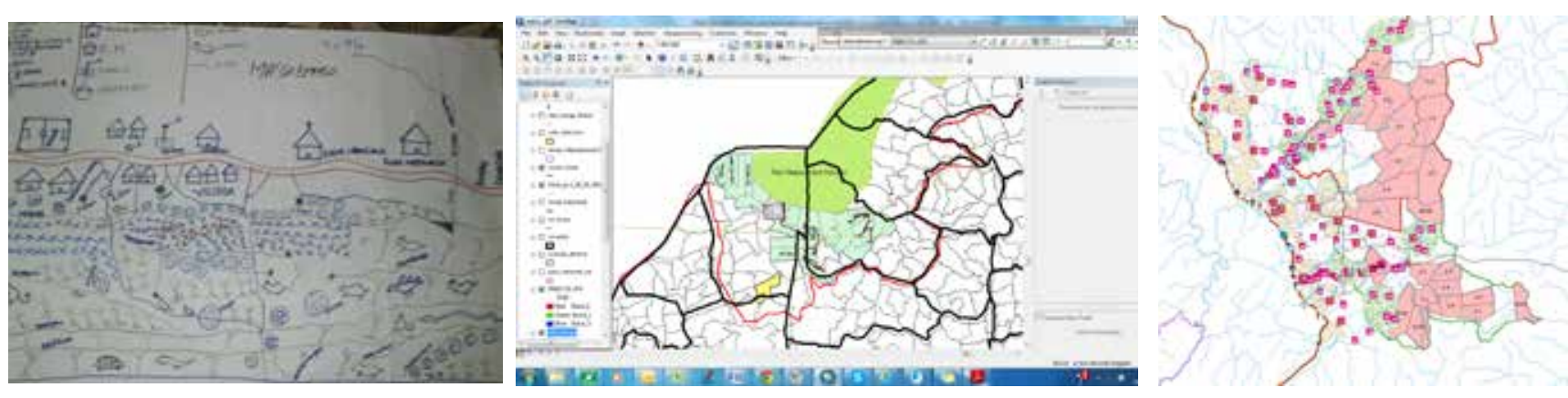

Figure 6. Carte participative sur papier ( $5 a)$, intégration des premières données dans le SIG (5b) et le rendu après traitement et intégration des données dans un SIG (5c)

sociaux doivent à l'aide du GPS enregistrer sur le terrain les pistes, les sites sacrés, les zones de cueillette, les zones de chasse. Munis du matériel de terrain (marqueurs, crayons, papier conférence), le facilitateur divise les équipes (selon le nombre de pistes et les zones d'intérêt) en donnant des informations sur les éléments à enregistrer (traces GPS terrain des pistes, limites et coordonnées GPS des centres d'intérêts).

Sur le terrain, le facilitateur ou le cartographe doit aider les cartographes/topographes locaux à se servir convenablement du GPS pour l'enregistrement des coordonnées géographiques et à remplir les fiches prévues à cet effet. Les photos des sites doivent être prises et associées aux coordonnées de la fiche d'enregistrement.
La carte finale est présentée aux villages et communautés voisines pour des réajustements et la validation.

\subsubsection{Numérisation des données}

A l'aide d'un logiciel SIG, la carte finale est réalisée. Les coordonnées GPS sont transférées dans le SIG, les éléments de la carte réalisée par les groupes de discussion sont intégrés dans le logiciel. Les éléments de la carte participative viennent s'intégrer dans ce système pour se rapprocher de la réalité de la géolocalisation. Les ajustements de la spatialisation sont un élément important de cette activité car c'est à travers cet exercice que tous les objets positionnés dans la carte participative se retrouveront aux endroits exacts sur le terrain, avec une marge d'erreur acceptable. 


\section{Encadré 3. Besoin de bien expliquer les objectifs de la CPGR}

Yokadouma est un site extrêmement sollicité par divers organismes dont ceux de conservation, de recherche, des exploitants forestiers, des exploitants miniers, des ONG de la société civile, etc. Cette sollicitation est due à la présence d'un massif forestier relativement bien conservé, partie de la Trinationale de la Sangha, érigé depuis 2012 en Patrimoine mondial de I'UNESCO pour sa richesse exceptionnelle en biodiversité faunique. De nombreux étudiants en provenance du monde entier y font également des recherches. Les populations sont donc habituées à recevoir des étrangers ayant des intérêts et des motivations divers.

Notre projet dans ce site porte sur la révision du plan simple de gestion de deux forêts communautaires. Les jeunes sélectionnés pour être formés à la réalisation de la cartographie participative ont exprimé des revendications au-delà de nos possibilités. Ils ont exigé d'être payés pour être formés à la cartographie en ces termes : « Vous allez d'abord nous payer avant d'exploiter nos forêts ». C'est à la suite de cette réaction que nous avons compris que nous étions confondus avec les exploitants forestiers.

Nous avons retenu que dans les sites où il y a pléthore d'interventions, il est important de bien expliquer non seulement l'approche, mais aussi et surtout les objectifs de l'exercice et les avantages que les populations pourront en tirer à plus ou moins long terme avant toute intervention.

Cette étape peut commencer sur le terrain et s'achever au bureau. La carte doit être réalisée avec minutie puisqu'il s'agit de représenter le vécu et l'imaginaire des populations. Les autres données topographiques et de télédétection doivent être utilisées afin de se rapprocher au plus près de la réalité et de conserver les échelles.

Une ébauche de la carte au format A1 ou A0 est produite pour validation sur le terrain avec les populations concernées.

\subsection{Phase 3 : Validation et finalisation de la carte}

La validation se fait sur le terrain, par toutes les composantes communautaires. C'est un exercice qui exige une grande vigilance parce qu'il peut générer des conflits entre des groupes sociaux. Dans les cas où un compromis n'a pu être trouvé, il convient de faire consigner les différents points de vue dans le rapport final.

\subsubsection{Validation}

La validation est un exercice qui consiste à présenter aux populations les cartes qui ont été faites par un groupe restreint et leur demander de commenter. Les commentaires ainsi faits permettent de constater si les participants comprennent ou non la carte proposée. Ils permettent aussi de corriger les erreurs, de compléter les informations manquantes, de faire une analyse des éléments de la carte ainsi que leur emplacement. Plusieurs copies des cartes sont imprimées en fonction du nombre de villages. Les personnes impliquées dans la réalisation de la carte doivent assister aux réunions de validation. Les réunions sont organisées dans les villages impliqués dans le processus. Les groupes socioculturels procèdent à la validation. Les ajouts sont enregistrés puis reportés sur la carte.

\subsubsection{Finalisation}

Au terme de ces travaux, les cartes corrigées et validées sont refaites dans le SIG, puis elles sont imprimées et distribuées à chaque communauté ainsi qu'aux autorités administratives et traditionnelles pour une utilisation éventuelle dans la gestion des ressources naturelles ou la reconnaissance des droits coutumiers. Elles pourront être affichées sur les lieux publics pour information.

\subsubsection{Le rapport final}

Un rapport global est dressé et annexé à la carte. Ce rapport comprend :

- les difficultés auxquelles les acteurs se sont confrontés ;

- les zones ou thématiques potentielles de conflits ;

- une vue d'ensemble sur les types d'utilisation des terres ( $\%$ ou superficies), la couverture forestière et les activités.

- les réponses aux questions de recherche. 
Tableau 2. Résultats attendus par étape

\begin{tabular}{|c|c|c|c|}
\hline Phases & Acteurs/compétences & Durée & Résultats attendus \\
\hline $\begin{array}{l}\text { Préparation au } \\
\text { bureau }\end{array}$ & $\begin{array}{l}\text { Cartographe, sociologue, } \\
\text { Socio-économiste, } \\
\text { Facilitateur, }\end{array}$ & Une semaine & $\begin{array}{l}\text { - Les premières cartes de base } \\
\text { sont produites } \\
\text { - Le guide méthodologique est } \\
\text { prêt } \\
\text { - Les rendez-vous et les } \\
\text { calendriers sont ajustés. }\end{array}$ \\
\hline Préparation sur le site & $\begin{array}{l}\text { Facilitateur (cartographe ou } \\
\text { sociologue) } \\
\text { Chef coutumier, } \\
\text { Administration, } \\
\text { Leader } \\
\text { ONG }\end{array}$ & $\begin{array}{l}1 \text { à } 2 \text { jours selon le } \\
\text { nombre de villages } \\
\text { impliqués et l'étendue du } \\
\text { projet }\end{array}$ & $\begin{array}{l}\text { - Les populations et les leaders } \\
\text { de projets sont sensibilisés } \\
\text { aux objectifs du projet et à la } \\
\text { nécessité de la réalisation de } \\
\text { la cartographie participative } \\
\text { - Les cartographes locaux sont } \\
\text { formés }\end{array}$ \\
\hline $\begin{array}{l}\text { Collecte des données } \\
\text { au GPS }\end{array}$ & $\begin{array}{l}\text { Cartographe, } \\
\text { Cartographes locaux, } \\
\text { Représentant de } \\
\text { l'administration, } \\
\text { Toutes les couches de la } \\
\text { population }\end{array}$ & 1 jour ou 2 jours & $\begin{array}{l}\text { La carte participative de chaque } \\
\text { village est réalisée }\end{array}$ \\
\hline $\begin{array}{l}\text { Numérisation des } \\
\text { données }\end{array}$ & Cartographe & 2 jours & $\begin{array}{l}\text { La première ébauche de la carte } \\
\text { est produite et imprimée sur } \\
\text { format } A 0\end{array}$ \\
\hline Validation & $\begin{array}{l}\text { Cartographe } \\
\text { Cartographes locaux } \\
\text { Représentant de } \\
\text { l'administration } \\
\text { Toutes les couches de la } \\
\text { population }\end{array}$ & 1 jour & $\begin{array}{l}\text { La carte est validée par les } \\
\text { populations locales }\end{array}$ \\
\hline Rapport & $\begin{array}{l}\text { Cartographe, socio- } \\
\text { économiste, sociologue }\end{array}$ & 2 jours & $\begin{array}{l}\text { La carte finale est produite et } \\
\text { distribuée }\end{array}$ \\
\hline
\end{tabular}




\section{Les acteurs et le materiel}

\subsection{Les acteurs de la CPGR}

La prise en compte des populations locales/ autochtones dans la prise de décisions concernant les espaces et les ressources dont elles partagent les droits est non seulement conforme à l'éthique, mais aussi est une exigence de la communauté internationale (FIDA, 2009). La cartographie participative est utilisée par les organismes de la communauté de recherche, les ONG internationales, les gouvernements et les sociétés privées comme porte d'entrée à cette participation.

Une cartographie participative géoréférencée fait appel à une équipe d'experts, des cartographes locaux, l'autorité administrative ainsi que la communauté locale, elle-même constituée par des institutions locales et des représentants des différents groupes sociaux.

\subsubsection{L'équipe des experts}

Une équipe d'experts facilite la réalisation de la carte de bout en bout. Elle comporte un ou une cartographe, un ou une anthropologue/sociologue et un ou une socio-économiste.

- Le cartographe est la cheville ouvrière de l'ensemble du processus de cartographie. À ce titre, il facilite la préparation, la réalisation et la finalisation de la CPGR. En amont, il organise une ou plusieurs rencontres avec les autorités administratives et traditionnelles pour leur expliquer la pertinence de l'exercice et recueillir leur assentiment. Il établit les critères de choix des individus qui seront formés à la cartographie et à l'utilisation des outils; Il supervise la formation des cartographes et techniciens locaux, puis le recensement des coordonnées géographiques et des traces des différents sites d'intérêt sur le terrain, enfin la finalisation et la validation des cartes.

- L'anthropologue/sociologue, tout comme le socio-économiste, assure la sensibilisation des populations avant et pendant la réalisation de la carte, l'organisation des groupes de discussion et la facilitation des discussions sur certains sujets en lien avec sa discipline. Il peut aussi s'occuper de la prise de notes sur les questions de recherche.

- Les cartographes locaux sont choisis dans leur communauté. Ce sont des hommes et des femmes, jeunes de préférence, dotés en plus d'un niveau d'instruction minimum requis (du fait de la formation théorique). Les cartographes locaux seront choisis en fonction d'un certain nombre de critères parmi lesquels la disponibilité, le sens du partage, la connaissance avérée de la vie au village et au-delà, des limites du terroir ou du village. Ce sont des intermédiaires entre les experts et les communautés. Ils assurent la liaison entre le village et l'extérieur dans la mesure où ils doivent ramener au village les informations et les formations reçues et renseigner les agents externes sur les préoccupations des populations. Leur travail consiste à utiliser le GPS pour enregistrer les coordonnées des lieux présentant un quelconque intérêt pour différents groupes sociaux et de participer à la réalisation, puis à la validation de la carte finale.

\subsubsection{L'autorité administrative}

Lautorité administrative est constituée d'un(e) responsable de l'administration locale garant(e) de la validité de la carte, d'un(e) représentant(e) du ministère en charge des forêts, d'un(e) représentant(e) du ministère en charge de l'agriculture et d'un(e) représentant(e) du ministère en charge des mines. Leur intervention est primordiale pour délimiter les espaces et participer à la validation de la carte définitive.

\subsubsection{La communauté locale}

La communauté locale comprend :

- Le chef du village ou de la communauté. Il doit participer à la réalisation de la carte, donner son avis sur la disposition des sites, participer à la validation de la carte finale.

- Les chefs religieux. Ils doivent non seulement participer la réalisation de la carte mais aussi donner leur point de vue en particulier sur les zones d'intérêt (lieux de culte, sites sacrés).

- Les représentants des corps de métiers. Ils doivent déterminer les zones de chasse, de pêche, d'agriculture, de mines artisanales et participer à l'élaboration et à la validation de la carte.

- Les groupes marginalisés. Ils doivent identifier leurs centres d'intérêt et participer à l'élaboration et la validation de la carte. 


\section{Encadré 4: Se préparer à gérer les conflits}

La réalisation des cartes sur le terrain est un exercice délicat, potentiellement conflictuel dans la mesure où les communautés peuvent la percevoir comme étant la confirmation administrative des limites géographiques des différents terroirs. A Nyabihu au Rwanda, à Lukolela en RDC et à Yokadouma au Cameroun, les chefs de village ou des cellules se sont empressés à placer sur la carte les limites de leurs villages respectifs, dans un esprit de positionnement. Dans certains cas, les limites des uns empiétaient sur les villages ou les espaces des autres. Les tensions latentes réveillées par cet exercice pouvaient à la longue se retourner contre l'équipe de la cartographie qui pouvait être indexée comme source du malheur.

Cette situation souligne la nécessité pour l'équipe de CPGR d'avoir des aptitudes à gérer les conflits. Dans tous les cas, le technicien ou les cartographes doivent rester neutres. L'équipe de CPGR se donne une meilleure chance en formalisant sa présence sur le terrain par une visite de courtoisie aux différentes autorités politicoadministratives et aux forces de l'ordre dès son entrée dans le site.

Le remboursement des frais de transport peut aussi générer des conflits entre les participants. Il convient de tenir compte des distances et du rôle des différents acteurs qui ont participé à la réalisation de la carte.

\subsection{Matériel et outils}

Le matériel est l'ensemble des objets physiques qui sert à une entreprise. Les outils représentent les instruments physiques ou non, utiles à la réalisation d'un ouvrage. Divers logiciels et techniques sont utilisés pour faciliter la réalisation de la carte : des SIG qui peuvent être gratuits (QGIS) ou payants (Arcgis, Erdas imagine), des GPS, des images satellites (landsat, Spot ou Rapideyes), des photographies aériennes prises à partir des avions (PGIS 05, 2005).

Il s'agit :

- Des logiciels SIG et de télédétection qui servent non seulement à la réalisation des cartes préliminaires et des cartes finales, mais aussi à la numérisation des données de terrain et au traitement des images satellites disponibles.

- Des cartes topographiques, des images satellites, des photographies aériennes qui sont nécessaires à la connaissance de la zone d'étude et à l'identification des éléments naturels.

- Du résumé des projets qui seront utilisés dans la sensibilisation des parties prenantes sur les activités qui seront implémentées.

- D'une liste des questions complémentaires à poser en vue d'approfondir les connaissances (voir en annexe).

Divers matériels et outils sont utilisés à différentes phases du processus. Le tableau 1 fait un récapitulatif de ces phases, des matériels et outils correspondants.
Sans être exhaustif, les objets suivants sont nécessaires à la réalisation de la CPGR :

- Un ordinateur, portable de préférence, qui servira à la réalisation des cartes ;

- Un vidéoprojecteur utilisé pour la projection des documentaires de sensibilisation et de formation ;

- Une boussole pour indiquer les directions et se repérer sur le terrain ;

- Un ou plusieurs GPS (Global positioning system), système de radio-navigation mondiale qui permet de calculer avec précision les coordonnées des points en $\mathrm{X}, \mathrm{Y}$ et $\mathrm{Z}$. Il servira à enregistrer les coordonnées géographiques des centres d'intérêt mais aussi à consigner les traces des routes et pistes.

- Le papier kraft, le papier conférence blanc et transparent;

- Des marqueurs/crayons-feutres sont utilisés pour dessiner sur les papiers conférence ;

- Les lampes torches et les tentes seront utiles au cas où l'équipe serait appelée à camper en forêt.

- Le kit de secours qui contient le matériel de premiers secours servira en cas de blessures légères ou de morsures.

- Un appareil photographique ou une caméra permettra de filmer les sites importants et les étapes jugées importantes.

- Des Fiches de collecte des données serviront à noter les caractéristiques des milieux et à enregistrer toutes informations utiles.

- Les machettes pour le défrichage.

NB. Les populations n'utiliseront pas les logiciels SIG mais seront associées au référencement au GPS. 
Tableau 3. Liste détaillée du matériel de travail

\begin{tabular}{|c|c|c|}
\hline $\mathbf{N}^{\circ}$ & Activités & Matériel/outils \\
\hline \multirow[t]{3}{*}{1} & \multirow{3}{*}{$\begin{array}{l}\text { Réalisation de la carte préliminaire } \\
\text { au bureau }\end{array}$} & Logiciel SIG et de télédétection \\
\hline & & Images satellites, carte topo, photo aérienne, textes et décrets \\
\hline & & $\begin{array}{l}\text { Ordinateurs (core } 15,500 \mathrm{Go} \text { de DD, RAM de } 8 \mathrm{GO} \text {, processeur } 2.6 \mathrm{Ghz} \times 2 \\
\text { ordinateurs portables de préférence en raison de leur mobilité) }\end{array}$ \\
\hline 2 & $\begin{array}{l}\text { Sensibilisation sur le terrain et } \\
\text { préparation participative de la } \\
\text { première ébauche de la carte. }\end{array}$ & $\begin{array}{l}\text { Flyer du Projet pilote, Vidéoprojecteur, Boussole, GPS, papier conférence } \\
\text { blanc et transparent, marqueurs }\end{array}$ \\
\hline 3 & Collecte des données GPS & $\begin{array}{l}\text { Ordinateur portable, papier conférence, marqueur, bloc note, } \\
\text { GPS, boussole, machettes, stylo, lampe torche, tente, kit de secours, }\end{array}$ \\
\hline 4 & Numérisation de la carte & $\begin{array}{l}\text { Ordinateur portable, logiciel libres (Qgis 2.0) et payants (Arcgis 10.1), } \\
\text { cartes, images satellitaires }\end{array}$ \\
\hline 5 & Restitution & Carte finale, matériel de bureau \\
\hline
\end{tabular}




\section{Coût de la cartographie participative géoréférencée}

Il est difficile d'évaluer le coût d'une CPGR. Il dépend de plusieurs facteurs dont la durée de l'exercice qui dépend à son tour de l'étendue du site à cartographier, de l'accessibilité du site et des éléments à cartographier, du degré de détail recherché, etc.

Dans tous les cas, les dépenses seront essentiellement de trois types : achat du matériel, main-d'œuvre et déplacement.

\subsection{Matériel}

Le matériel requis a déjà été évoqué plus haut. Les prix varient en fonction des pays. Le tableau 4 donne un exemple de matériel requis pour la cartographie d'un village au Cameroun. Il ne prend pas en compte l'achat des logiciels, du GPS, de la boussole et de l'ensemble des outils de numérisation.

Les administrations nationales en charge des forêts peuvent apporter des contributions qui sont de nature à réduire considérablement les dépenses. À titre d'exemple, les images Spot et les shapefiles de Lukoléla, Nyabihu et Yokadouma ont été gracieusement offerts par les administrations en charge des forêts de la RDC, du Rwanda et du Cameroun, respectivement. Autrement, il aurait fallu prévoir environ 2000 dollars par site pour leur achat.

Tableau 4. Elément de coût du matériel de terrain pour la réalisation d'une carte participative géoréférencée

\begin{tabular}{lccr}
\hline Matériel & Quantité & $\begin{array}{c}\text { Prix unitaire } \\
\text { (USD) }\end{array}$ & $\begin{array}{r}\text { Total } \\
\text { (USD) }\end{array}$ \\
\hline Boussole et GPS & $/$ & $/$ & $/$ \\
$\begin{array}{l}\text { Papier conférence } \\
\text { ou papier Kraft }\end{array}$ & 1 & 40 & 40 \\
Marqueur & 20 & 1 & 20 \\
Kit de secours & 2 & 100 & 200 \\
Lampe torche & 10 & 3 & 30 \\
Machette et limes & 10 & 6 & 60 \\
Matériel de bureau & 1 & 40 & 40 \\
Total & & & 390 \\
Imprévus & & $10 \%$ & 39 \\
Total & & & 390 \\
\hline
\end{tabular}

Si les villages sont loin des sites à cartographier, il faut prévoir des tentes en vue de camper en forêt.

\subsection{La main d'œuvre}

Lors du calcul des coûts de la main d'œuvre, il faudra prendre en compte les experts extérieurs qui peuvent être appelés à intervenir dans l'exercice. Ces coûts dépendent de la durée de la mission. Par ailleurs, les personnes mobilisées dans les villages pour la cartographie reçoivent une compensation, qui varie en fonction de leur rôle d'une part, du site d'autre part. Elle est fonction de plusieurs facteurs dont la capacité de négociation des acteurs et les perceptions du projet par les populations locales. Celles-ci sont moins exigeantes pour les projets de développement portés par les ONGs locales. À titre d'exemple, à Yokadouma au Cameroun, les cartographes locaux ont reçu $8 \$ /$ jour/personne et les accompagnateurs, $4 \$$ tandis qu'à Nyabihu au Rwanda, les premiers ont touché $21 \$$ et les seconds, $10 \$$.

\subsection{Le déplacement}

Le déplacement dont il est question désigne indistinctement le voyage de l'équipe de chercheurs de leur lieu de travail jusque dans le site, mais aussi le déplacement à l'intérieur des sites. Si le coût du premier est relativement aisé à déterminer, celui du second est une véritable gageure, dans la mesure où il dépend de facteurs difficilement maîtrisables par les experts vivant souvent loin des sites. Ces facteurs peuvent être l'accessibilité du site, le niveau de développement des infrastructures, l'étendue du site, etc. Plusieurs moyens de déplacement pourront être mis à contribution : à pied, à vélo, à moto, en pirogue ou en voiture.

Dans le site de Lukolela en RDC par exemple, la location d'une pirogue motorisée est revenue à US\$120 par jour, incluant les honoraires du pinassier ${ }^{1}$, le carburant, le moteur et la pirogue. À Nyabihu il a fallu US $\$ 30$ pour la location d'une motocyclette, et US\$20 à Yokadouma. Globalement, le processus de réalisation de la CPGR prévoit

1 Le conducteur de la pirogue. 
Tableau 5. Dépenses effectuées lors de la phase terrain de la réalisation de la cartographie participative géoréférencée dans trois sites.

\begin{tabular}{|c|c|c|c|c|c|}
\hline Etapes & Activités & $\begin{array}{l}\text { Coût à Lukoléla } \\
\text { RDC (US\$) }\end{array}$ & $\begin{array}{l}\text { Coût à Nyabihu } \\
\text { Rwanda (US\$) }\end{array}$ & $\begin{array}{l}\text { Coût à Yokadouma } \\
\text { Cameroun (US\$) }\end{array}$ & Observations \\
\hline & & 20 villages & 20 cellules & 7 villages & \\
\hline & & $32255 \mathrm{ha}^{\mathrm{a}}$ & 9648,25 ha & 10000 ha & \\
\hline & & $\begin{array}{l}\text { Accès par fleuves, } \\
\text { mauvais état des } \\
\text { routes }\end{array}$ & $\begin{array}{l}\text { Accès par route } \\
\text { bitumées, routes } \\
\text { non entretenues }\end{array}$ & $\begin{array}{l}\text { Accès par routes } \\
\text { non bitumées } \\
\text { Routes non }\end{array}$ & \\
\hline & & Moyen de & Moyen de & entretenues & \\
\hline & & $\begin{array}{l}\text { déplacement: } \\
\text { Pirogue, moto }\end{array}$ & $\begin{array}{l}\text { déplacement : } \\
\text { véhicules et motos }\end{array}$ & $\begin{array}{l}\text { Moyen de } \\
\text { déplacement: }\end{array}$ & \\
\hline & & Faible densité de & Forte densité de & véhicules et motos & \\
\hline & & population & population $(456,1$ & Faible densité de & \\
\hline & & $\left(32,2 \mathrm{~h} . / \mathrm{Km}^{2}\right)$ & $\mathrm{h} / \mathrm{km}^{2}$ ) & $\begin{array}{l}\text { population }(40,2 \mathrm{~h} / \\
\left.\mathrm{km}^{2}\right)\end{array}$ & \\
\hline $\begin{array}{l}\text { Sensibilisation } \\
\text { des autorités } \\
\text { (par site) }\end{array}$ & $\begin{array}{l}\text { Rencontre } \\
\text { individuelles } \\
\text { avec les }\end{array}$ & $\begin{array}{l}100 \text { US\$ pour } 5 \\
\text { personnalités } \\
\text { dont chef de cité, }\end{array}$ & $\begin{array}{l}25 \text { US\$ pour } 3 \\
\text { personnalités dont } \\
\text { le maire, les chefs }\end{array}$ & $\begin{array}{l}100 \text { US\$ pour } 5 \\
\text { personnalités } \\
\text { (MINAT, MINFOF, }\end{array}$ & $\begin{array}{l}\text { Les montants } \\
\text { énumérés } \\
\text { incluent la }\end{array}$ \\
\hline $\begin{array}{l}\text { Durée : une } \\
\text { demi-journée }\end{array}$ & $\begin{array}{l}\text { autorités } \\
\text { administratives }\end{array}$ & $\begin{array}{l}\text { chef de secteur, } \\
\text { administration } \\
\text { déconcentrée }\end{array}$ & de secteurs) & Forces de l'ordre) & $\begin{array}{l}\text { nutrition et le } \\
\text { transport }\end{array}$ \\
\hline $\begin{array}{l}\text { Sensibilisation } \\
\text { des } \\
\text { communautés } \\
\text { (par village) }\end{array}$ & $\begin{array}{l}\text { Réunion de } \\
\text { sensibilisation } \\
\text { avec les } \\
\text { autorités } \\
\text { traditionnelles }\end{array}$ & $\begin{array}{l}50 \text { US\$ pour } 5 \\
\text { personnes }\end{array}$ & $\begin{array}{l}30 \text { US\$ pour } 3 \\
\text { personnes par } \\
\text { cellule }\end{array}$ & $\begin{array}{l}50 \text { US\$ pour } 5 \\
\text { personnes }\end{array}$ & $\begin{array}{l}\text { Essentiellement } \\
\text { pour } \\
\text { déplacement } \\
\text { et alimentation } \\
\text { durant les }\end{array}$ \\
\hline $\begin{array}{l}\text { demi-journée } \\
\text { par village }\end{array}$ & $\begin{array}{l}\text { Sélection des } \\
\text { cartographes } \\
\text { et personnes } \\
\text { ressources }\end{array}$ & & & & rencontres \\
\hline $\begin{array}{l}\text { Formation des } \\
\text { cartographes }\end{array}$ & $\begin{array}{l}\text { Formation à } \\
\text { la réalisation }\end{array}$ & $\begin{array}{l}360 \text { US \$ pour } 30 \\
\text { participants soit } 12\end{array}$ & $\begin{array}{l}510 \text { US\$ pour } 30 \\
\text { cartographes }\end{array}$ & $\begin{array}{l}120 \text { US\$ pour } 15 \\
\text { participants soit }\end{array}$ & $\begin{array}{l}\text { Pour } \\
\text { déplacement et }\end{array}$ \\
\hline $\begin{array}{l}\text { locaux (par } \\
\text { site) }\end{array}$ & $\begin{array}{l}\text { de la carte } \\
\text { participative et }\end{array}$ & US\$/ participant & $\begin{array}{l}\text { Soit 17US\$/ } \\
\text { participant }\end{array}$ & $8 \$ /$ participants & $\begin{array}{l}\text { alimentation des } \\
\text { cartographes }\end{array}$ \\
\hline $\begin{array}{l}\text { Durée : une } \\
\text { Journée }\end{array}$ & $\begin{array}{l}\text { à l'utilisation du } \\
\text { GPS. }\end{array}$ & & & & $\begin{array}{l}\text { et personnes } \\
\text { ressources }\end{array}$ \\
\hline $\begin{array}{l}\text { Prise des } \\
\text { coordonnées } \\
\text { (par village) }\end{array}$ & $\begin{array}{l}\text { Prise des } \\
\text { coordonnées } \\
\text { des sites }\end{array}$ & $\begin{array}{l}30 \text { US\$ pour } 4 \\
\text { personnes, } 12 \text { US\$ } \\
\text { pour l'opérateur GPS }\end{array}$ & $\begin{array}{l}41 \text { US\$ pour } 3 \\
\text { personnes } 21 \text { US\$ } \\
\text { pour l'opérateur }\end{array}$ & $\begin{array}{l}16 \text { US\$ pour } 3 \\
\text { personnes, } 8 \text { US\$ } \\
\text { pour l'opérateur }\end{array}$ & $\begin{array}{l}\text { Les montants } \\
\text { énumérés } \\
\text { incluent }\end{array}$ \\
\hline $\begin{array}{l}\text { Durée : une } \\
\text { demi-journée }\end{array}$ & $\begin{array}{l}\text { d'intérêts et } \\
\text { les traces, } \\
\text { les routes, } \\
\text { les pistes, les } \\
\text { infrastructures, } \\
\text { etc. pour les } \\
\text { communautés }\end{array}$ & $\begin{array}{l}\text { et } 6 \text { US\$ pour les } \\
\text { accompagnateurs }\end{array}$ & $\begin{array}{l}\text { et } 10 \text { US\$ pour les } \\
\text { accompagnateurs }\end{array}$ & $\begin{array}{l}\text { et } 4 \text { US\$ pour les } \\
\text { accompagnateurs }\end{array}$ & $\begin{array}{l}\text { l'alimentation et } \\
\text { le transport. }\end{array}$ \\
\hline $\begin{array}{l}\text { Réalisation } \\
\text { de la carte }\end{array}$ & $\begin{array}{l}\text {-Dessin des } \\
\text { infrastructures, }\end{array}$ & $\begin{array}{l}108 \text { US\$ pour } 13 \\
\text { personnes, } 12\end{array}$ & $\begin{array}{l}142 \text { US\$ pour } 12 \\
\text { personnes, } 21\end{array}$ & $\begin{array}{l}56 \text { US\$ pour } 12 \\
\text { personnes, } 8\end{array}$ & $\begin{array}{l}\text { Les montants } \\
\text { énumérés }\end{array}$ \\
\hline $\begin{array}{l}\text { participative } \\
\text { (par village) }\end{array}$ & $\begin{array}{l}\text {-Positionnement } \\
\text { des types de }\end{array}$ & $\begin{array}{l}\text { US\$ pour les } 3 \\
\text { cartographes et }\end{array}$ & $\begin{array}{l}\text { US\$ pour les } 2 \\
\text { cartographes et }\end{array}$ & $\begin{array}{l}\text { US\$ pour les } 2 \\
\text { cartographes et }\end{array}$ & $\begin{array}{l}\text { incluent } \\
\text { l'alimentation et }\end{array}$ \\
\hline $\begin{array}{l}\text { Durée : une } \\
\text { demi-journée }\end{array}$ & LULC & $\begin{array}{l}6 \text { US\$ pour les } \\
\text { participants }\end{array}$ & $\begin{array}{l}10 \text { US\$ pour les } \\
\text { participants }\end{array}$ & $\begin{array}{l}4 \text { US\$ pour les } \\
\text { participants }\end{array}$ & le transport \\
\hline Total & & 648 & 738 & 342 & \\
\hline
\end{tabular}

a Sur cette superficie, seulement 4179 ha seront considérés comme zone où les activités seront implémentées 


\section{Encadré 5 : Budgets des activités}

Bien que les rubriques soient les mêmes, les dépenses varient considérablement d'un site à un autre. En RDC, le transport des cartographes locaux jusque dans les villages a explosé les budgets. Les déplacements dans les villages Maberu, Mpumba, Mboko, Malilo se sont effectués à l'aide de pirogues motorisées qu'il fallait louer. De plus, il a fallu payer des hébergements aux équipes qui ne pouvaient pas faire des va-et-vient. Toutes des rubrique qui n'avaient pas été prévues.

Au Rwanda, en plus des dépenses liées au transport, le relief très accidenté et la forte densité des populations ont ralenti considérablement les équipes, rallongeant ainsi les montants à payer. Le niveau de vie du pays peut influencer considérablement le budget : la plupart des coûts sont passés du simple en RDC au double au Rwanda.

L'élaboration des budgets pour la réalisation de l'activité de cartographie participative doit tenir compte des réalités de terrain. Pour cela il est important de connaître les prix usuels en ce qui concerne le transport, le niveau de vie, la nutrition, l'hébergement, les salaires, le logement au quotidien. Lorsque les investigations ne sont pas bien menées pour obtenir des précisions sur ces informations, les conséquences peuvent être désastreuses.

trois descentes sur les sites, la première pour la sensibilisation, la seconde pour la réalisation de la carte participative et la dernière pour la validation de la carte géoréférencée. Toutes ces descentes impliquent des coûts qu'il faut évaluer soigneusement. Le tableau 5 présente les dépenses qui ont été effectuées lors de la phase terrain de la réalisation de la cartographie participative géoréférencée dans trois sites. 


\section{Conclusion}

La CPGR présentée dans ce document permet de représenter le terroir en projetant chaque objet à la place requise. La réalisation des cartes passe par trois phases qui se déroulent entre le bureau et le terrain. Il ressort des expériences que le déroulement de l'exercice peut être mis à mal par plusieurs difficultés parmi lesquelles les concordances budgétaires, la gestion des conflits ou la mobilisation des acteurs.
La budgétisation des activités doit tenir compte du contexte du milieu et du pays, des distances à parcourir dans les sites et surtout du comportement des acteurs concernés. Les objectifs de la CPGR, les rôles et les responsabilités des experts, des cartographes locaux et des communautés doivent être clairement définis et expliqués avant le lancement des activités sur le terrain. 


\section{Bibliographie}

Amelot, X. 2013. Cartographie participative pour le développement local et la gestion de l'environnement à Madagascar, empowerment, impérialisme numérique ou illusion participative? L'Information géographique, 77(4), 47-67.

Anau, N., Corbett, J., Iwan, R., van Heist, M., Limberg, G., Sudana, M., Wollenberg, E. 2003. Do communities need to be good mapmakers? Centre de recherche forestière internationale, Jakarta, Indonésie, Téléchargé à partir de http:// www.iapad-org

Angeon, V., Caron P., Lardon S. 2006. "Des liens sociaux à la construction d'un développement territorial durable: quel est le rôle de la proximité dans ce processus? Développement durable et territoires [En ligne], Dossier 7 | 2006, mis en ligne le 10 mai 2006, consulté le 19 août 2014. URL : http://developpementdurable.revues. org/2851;DOI :10.4000/developpement durable. 285

Burini F. 2005. La cartographie participative dans la recherche de terrain en Afrique : le cas des villages riverains au Parc Régional W, dans: Policy Matters, special edition W Regional Park, IUCN, publié en ligne: http://www.iapad.org/ biblio/2005.htm.

Burini, F. 2009. La cartographie participative et la pratique du terrain dans la coopération environnementale : la restitution des savoirs traditionnels des villages de l'Afrique subsaharienne, dans Colloque À travers l'espace de la méthode : les dimensions du terrain en géographie, 18-20 juin 2008, Arras, France [halshs-00389595, version 1 - 29/05/2009].

Burini, F. 2013. L'évolution de la cartographie auprès des sociétés traditionnelles en Afrique subsaharienne. L'Information géographique. Edition Armand Colin 77(4): 68-87.

Chambers, R. 2006. Participatory mapping and geographic information systems: whose map? Who is empowered and who disempowered? Who gains and who loses? The Electronic Journal of Information Systems in Developing Countries 25 (2), 1-11.

Chapin, M., Lamb, Z., \& Threlkeld, B. 2005. Mapping indigenous lands. Annu. Rev. Anthropol., 34, 619-638.
Corbett, J. and Rambaldi G. 2009. Representing our reality: Geographic information technologies, local knowledge and change, Cope, M.\& Elvood, S. (Eds.), Qualitative GIS: Mixed methods in practice and theory, 2009

Denniston, D. 1994. Defending the land with maps. World watch 7(1): 27-31.

Di Gessa, S. 2008. Participatory mapping as a tool for empowerment: Experiences and lessons learned from the ILC network. Rome: ILC/ IFAD 53p.

FIDA, 2009. Cartographie participative et bonnes pratiques, FIDA, Rome. Italie, 59p

FIDA, 2010. cartographie participative: l'approche évolutive de la FIDA, FIDA, Rome. Italie 47p

Flavelle, A. 2002. Mapping our land: A Guide to Making Your Own Maps of Communities \& Traditional Lands. Edmonton, AB:Lone pine Foundation 204p.

Hervé D., Ramaroson J.H, Randrianarison A. et Le Ber F. 2014. Comment les paysans du corridor forestier de Fianarantsoa (Madagascar) dessinent-ils leur territoire ? Des cartes individuelles pour confronter les points de vue, Cybergeo : European Journal of Geography [En ligne], Cartographie, Imagerie, SIG, document 681, mis en ligne le 23 juillet 2014, consulté le 18 août 2014. URL : http:// cybergeo.revues.org/26387 ; DOI : 10.4000/ cybergeo. 26387

Jardinet, S. 2006. Capacity development and PGIS for land demarcation: innovations from Nicaragua. Participatory Learning and Action 54(1): 67-73.

Kwaku Kyem, P. A. 2004. Of intractable conflicts and participatory GIS applications: The search for consensus amidst competing claims and institutional demands. Annals of the Association of American Geographers 94(1): 37-57.

Long., Georges Handja T. et Gata T. 2013; la cartographie participative: guide pour la production des cartes avec les communautés forestières dans le bassin du congo; The Rainforest Foundation UK, London, UK 11P

Momberg, F., Damus, D., Limberg, G., \& Padan, S. 1994. Participatory tools for communityforest profiling and zonation of conservation areas: Experiences from the Kayan Mentarang 
Nature Reserve, East Kalimantan, Indonesia. Washington DC, WWF Indonesia Program; Kayan mentarang Project (PHPA-WWF-LIPI)

PALSKY, G. 2010. Cartes participatives, cartes collaboratives: La cartographie comme maïeutique. Le Monde des cartes (205): 49-59.
Rambaldi, G. 2005. Who owns the map legend? URISA-WASHINGTON DC- 17(1): 5.

PGIS ‘05 - 2005. Mapping for Change International Conference on Participatory Spatial Information Management and Communication . cta.int KCCT Nairobi, Kenya, consulté le 20 Avril 2014. http://pgis-tk-en.cta.int/m05/index.html 


\section{Annexe 1: Guide d'enquête}

\begin{tabular}{|c|c|c|c|}
\hline Objectif & \multicolumn{3}{|c|}{ Obtenir une carte de la situation actuelle de la zone du projet et une cartographie des risques. } \\
\hline \multirow[t]{7}{*}{ Déroulement } & \multicolumn{3}{|c|}{ Essayer d'impliquer autant que possible les participants dans l'ébauche de la carte. } \\
\hline & \multicolumn{3}{|l|}{ Commencer avec des références claires: } \\
\hline & \multicolumn{3}{|l|}{ 1. Limites du village, limites du projet } \\
\hline & \multicolumn{3}{|c|}{ 2. Points de référence : mairie, maison du chef, église/mosquée, routes, fleuves. } \\
\hline & \multicolumn{3}{|c|}{ Marquer les autres éléments dans la liste ci-dessous. } \\
\hline & \multicolumn{3}{|l|}{ Discuter les questions d'analyse. } \\
\hline & \multicolumn{3}{|c|}{ 3. Prendre des notes pendant la discussion et une photo du résultat final. } \\
\hline \multirow{11}{*}{$\begin{array}{l}\text { Questions } \\
\text { d'analyse }\end{array}$} & Elément & Questions potentielles & Questions de discussion \\
\hline & $\begin{array}{l}\text { Lignes de base : lieu de la réunion, } \\
\text { routes, fleuves. }\end{array}$ & $\begin{array}{l}\text { Où sommes-nous situés ? Où sont les routes } \\
\text { principales? où sont les fleuves, rivières et } \\
\text { lacs? Marquer la distance. }\end{array}$ & $\begin{array}{l}\text { D'où vient l'eau? Buvez-vous l'eau venant des rivières? } \\
\text { La source d'eau est-elle aménagée pour avoir une bonne } \\
\text { qualité d'eau? }\end{array}$ \\
\hline & \multirow{4}{*}{$\begin{array}{l}\text { Limites : Paysage, projet, village, forêt } \\
\text { communautaire (si c'est le cas) et } \\
\text { zone de fuite. }\end{array}$} & $\begin{array}{l}\text { Où sont les limites de la forêt ? Est-elle privée, } \\
\text { partagée, communautaire ? (indiquer) }\end{array}$ & \multirow[t]{4}{*}{$\begin{array}{l}\text { S'il y a une forêt communautaire, est-ce qu'elle a des limites } \\
\text { physiques claires? }\end{array}$} \\
\hline & & $\begin{array}{l}\text { Où est-ce que les activités du projet auront } \\
\text { lieu? }\end{array}$ & \\
\hline & & $\begin{array}{l}\text { Quel est l'endroit le plus éloigné où les gens } \\
\text { collectent le bois de feu, et les PFNL? }\end{array}$ & \\
\hline & & $\begin{array}{l}\text { Où sont les champs ? Quelles sont les } \\
\text { principales cultures? }\end{array}$ & \\
\hline & $\begin{array}{l}\text { Infrastructure : provision et dépôt } \\
\text { d'eau, routes, maisons, services } \\
\text { sociaux (puits, écoles, hôpitaux, } \\
\text { marchés, mairie) }\end{array}$ & $\begin{array}{l}\text { Où sont les écoles, puits, marchés, églises/ } \\
\text { mosquées, irrigation, bureau administratif, etc. } \\
\text { ? autres bâtiments importants? }\end{array}$ & \\
\hline & \multirow{2}{*}{$\begin{array}{l}\text { Flore : champs, forêt par type } \\
\text { (primaire, secondaire) - privée, } \\
\text { communautaire, familles, etc. }\end{array}$} & Cultures principales dans chaque champ? & \multirow{4}{*}{$\begin{array}{l}\text { Où obtenez-vous le bois de construction ? de vente ? Où } \\
\text { collectez-vous le bois de feu? Produisez-vous du charbon } \\
\text { ? Où prenez-vous le bois pour produire du charbon? Et les } \\
\text { autres produits forestiers? Sont-ils pour la consommation } \\
\text { ou la vente? Sont-ils exportés ou importés (flèches)? Qui } \\
\text { collecte? }\end{array}$} \\
\hline & & $\begin{array}{l}\text { Quelles sont les arbres et plantes les plus } \\
\text { utilisés en forêt? Quel type de forêt entoure le }\end{array}$ & \\
\hline & \multirow{2}{*}{$\begin{array}{l}\text { Produits forestiers, agriculture, } \\
\text { déforestation. }\end{array}$} & village? & \\
\hline & & $\begin{array}{l}\text { Où l'agriculture sur brulis a-t-elle lieu? Et } \\
\text { l'exploitation du bois? La production de } \\
\text { charbon? }\end{array}$ & \\
\hline
\end{tabular}


Qui gère chaque partie de la forêt? (indiquer si des paysans individuels, des familles, des clans, la communauté, une compagnie, l'État). Comment la forêt est-elle gérée ? Quelles sont les principales activités/responsabilités des personnes en charge/institutions? (permis de coupe partage des terrains, gestion des conflits). Rôle des hommes, femmes et pygmées.

Expliquez dans quelle mesure il vous est permis de vous engager dans des activités productives ? Combien peut être collecté par la communauté ? Quoi ? Quand? Par qui?

Quels bénéfices économiques et de subsistance obtenezvous de chaque type de forêt?

Pensez-vous que le couvert forestier a diminué? Pourquoi ? Où?

Sol: Meilleur et pire terrain.

Utilisation des terres à émission de GES

Animaux et vie sauvage:

- Zones d'élevage et espèces

- Zones de chasse et espèces. (légale et illégale)

Cartographie des risques: risques et effets climatiques: vents (venant d'où?), inondations, érosion (voir la direction de la pluie quand il pleut)

Les zones à risques

Quels sont les meilleurs et les pires terrains?

(érosion, infertilité, sècheresse, etc.)

Y a-t-il des marécages ? Cultivez-vous là-bas ?

Quoi?

Où sont les principales zones de chasse? Quel type d'animaux chassez-vous? Qui chasse ? O le bétail est-il élevé et nourri?

Où subissez-vous les inondations? Y a-t-il des vents forts ? Où ? Le vent et l'eau détruisent-ils le sol, les maisons, etc. ?

Quelles sont les zones à risques dans le village ? Inondations, glissement de terrain?

\begin{tabular}{ll} 
Temps & 3 heures \\
Matériel & Papiers conférence et marqueurs \\
Légende & (symboles et signification) \\
\hline
\end{tabular}

Y a-t-il de l'agriculture dans des zones que le projet envisage protéger?

Y a-t-il des problèmes d'érosion ? D'inondation ? où? pourquoi?

Y a-t-il des animaux interdits? Selon vous qu'est-ce que le braconnage? Les animaux sont-ils plus ou moins abondants ? Où nourrissez-vous le bétail ? dans les champs? Dans une cabane? En forêt, Pourquoi?

D'où viennent les inondations (flèches)? Quel fleuve ? Et les vents (flèches) ? 


\section{Annexe 2 : Liste indicative des éléments à représenter dans la légende}

- Champ (cacao, café, vivrier, agro forêt)

- Jachère (moins de 10 ans, plus de 10 ans)

- Forêt secondaire

- Forêt primaire

- Habitat humain

- Habitat de la faune sauvage et espèces d'animaux emblématiques

- Infrastructures sociales (chefferie, dispensaire, école, lieu de culte, forage, puits, cimetière, site sacré ...)

- Infrastructures administratives (police, douane, préfecture, gendarmerie, bureau administration locale)

- Infrastructures économiques (usines, entreprises)

- Limite de villages

- Hydrographie (cours d'eau, sources, lac, étangs, marécages)

- Routes, pistes

- Zone de chasse, zone de pêche

- Zone à risques 

Les documents de travail du CIFOR contiennent des résultats préliminaires ou anticipés concernant d'importantes questions sur les forêts tropicales qui nécessitent d'être publiées à l'avance. Ces documents sont produits pour informer et inciter la discussion. Leur contenu a été revisé à l'interne, mais n'a pas subi la procédure d'examen par les pairs.

La connaissance des terroirs villageois et des types d'occupation des terres est primordiale pour réaliser les scénarios de référence dans le cadre des projets qui entendent bénéficier des payements pour services environnementaux. Dans ce contexte, la cartographie participative a fait ses preuves en tant qu'outil de facilitation de la participation populaire dans la mesure où elle permet aux populations locales de s'exprimer et de représenter les activités et les types d'utilisation des terres de leur terroir. Plus encore, la cartographie participative dite 'géoréférencée' permet non seulement de positionner les objets en relation avec les coordonnées spatiales (GPS) mais aussi de numériser ces données afin de ressortir un rendu validé et utilisable par toutes les parties prenantes. Si l'utilité de ce type d'outil est de plus en plus reconnue, des difficultés demeurent quant au protocole de sa réalisation.

Ce document présente les étapes de la réalisation de cette cartographie géoréférencée, assorties des leçons tirées des expériences acquises au Cameroun, en RDC et au Rwanda dans le cadre du projet COBAM (Changement Climatique et Forêt dans le Bassin du Congo : Synergie entre adaptation et atténuation) lancé par le CIFOR en 2010.

Ce guide est un aide-mémoire qui expose les différentes phases de la réalisation de la cartographie participative, puis de sa digitalisation. II propose des astuces utiles à la réussite du processus et donne un aperçu des coûts de certaines activités.

\begin{tabular}{|l|l|l|l} 
Cette recherche a été menée par le CIFOR dans le cadre du Programme de recherche du CGIAR \\
sur les forêts, les arbres et l'agroforesterie (CRP-FTA). Ce programme collaboratif vise à améliorer la \\
gestion et l'utilisation des forêts, de l'agroforesterie et des ressources génétiques des arbres à l'échelle \\
du paysage, des forêts aux exploitations agricoles. Le CIFOR dirige le CRP-FTA en partenariat avec \\
Bioversity International, le CIRAD, le CATIE, le Centre international d'agriculture tropicale et le Centre \\
mondial de l'Agroforestrie.
\end{tabular}

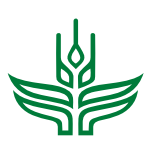

CGIAR
Fund

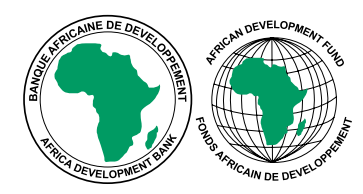

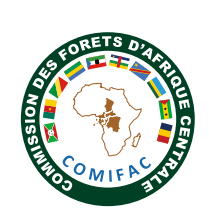

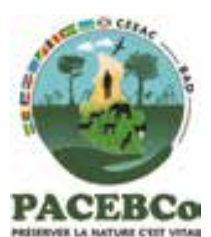

Centre de recherche forestière internationale (CIFOR)

Le CIFOR oeuvre en faveur du bien-être humain, de la conservation de l'environnement et de l'équité par sa recherche scientifique qui contribue à l'élaboration des politiques et des pratiques affectant les forêts dans les pays en développement. Le CIFOR est membre du Consortium du CGIAR. Son siège est situé à Bogor en Indonésie et il est également implanté en Asie, en Afrique et en Amérique latine. 